Title:

Robot Visual Control

Name: $\quad$ François Chaumette

Affil./Addr.: Inria, Univ Rennes, CNRS, IRISA

Encyclopedia of Systems and Control, 2nd edition

J. Baillieul, T. Samad (eds), Springer-Verlag, London, 2019

\title{
Robot Visual Control
}

\section{Abstract}

This entry presents the basic concepts of vision-based control, that is, the use of visual data to control the motions of a robotics system. It details the modeling steps allowing to achieve a visual task, the list of possible visual features that can be used as input of the control scheme, the design of basic kinematics control schemes, and hints towards more advanced approaches. Applications are also described.

\section{Keywords}

Robot control, robot vision, visual servoing, Jacobian

\section{Introduction}

Basically, visual control, also named visual servoing, consists in using the data provided by one or several cameras as input of real-time closed-loop control schemes for controlling the motions of a dynamic system so that it achieves a task specified by a set of visual constraints (Hutchinson et al. 1996; Chaumette et al. 2016). Such systems 
are usually robot arms or mobile robots, but can also be virtual robots, or even a virtual camera. A large variety of positioning tasks, or target tracking tasks, can be considered by controlling from one to all the degrees of freedom (DoF) of the system (Marchand et al. 2005). Whatever the sensor configuration, which can vary from one on-board camera located on the robot end-effector to several free-standing cameras, a set of visual features has to be selected at best from the available image measurements, allowing to control the desired DoF. A control law has then to be designed so that these visual features reach a desired value, defining a correct achievement of the task. A desired planned trajectory can also be tracked. The control principle is to regulate the error between the current and desired values of the visual features to zero, or, in other terms, to minimize an objective function from which Lyapunov-based stability analysis can be performed. With a vision sensor providing 2D measurements, potential visual features are numerous, since $2 \mathrm{D}$ data (coordinates of particular points in the image, parameters related to geometrical shapes, intensity levels of set of pixels,...) as well as 3D data provided by a localization algorithm exploiting the extracted 2D measurements can be considered.

Typically, an iteration of the control scheme consists of the following successive steps:

- $\quad$ acquire an image;

- $\quad$ extract some useful image measurements;

- compute the current value of the visual features used as inputs of the control scheme;

- compute the error between the current and the desired values of the visual features;

- update the control outputs, which are usually the robot velocity, to regulate that error to zero, i.e. to minimize its norm. 
For instance, for the first example depicted on Fig. 1, the image processing part consists in extracting and tracking the center of gravity of the moving people, the visual features are composed of the two Cartesian coordinates of this center of gravity, and the control scheme computes the camera pan and tilt velocities so that the center of gravity is as near as possible of the image center despite the unknown motion of the people. In the second example where a camera mounted on a six DoF robot arm is considered, the image measurements are a set of segments that are tracked in the image sequence. From these measurements and the knowledge of the 3D object model, the pose from the camera to the object is estimated and used as visual features. The control scheme now computes the six components of the robot velocity so that this pose reaches a particular desired value corresponding to the object position depicted in blue on the images.
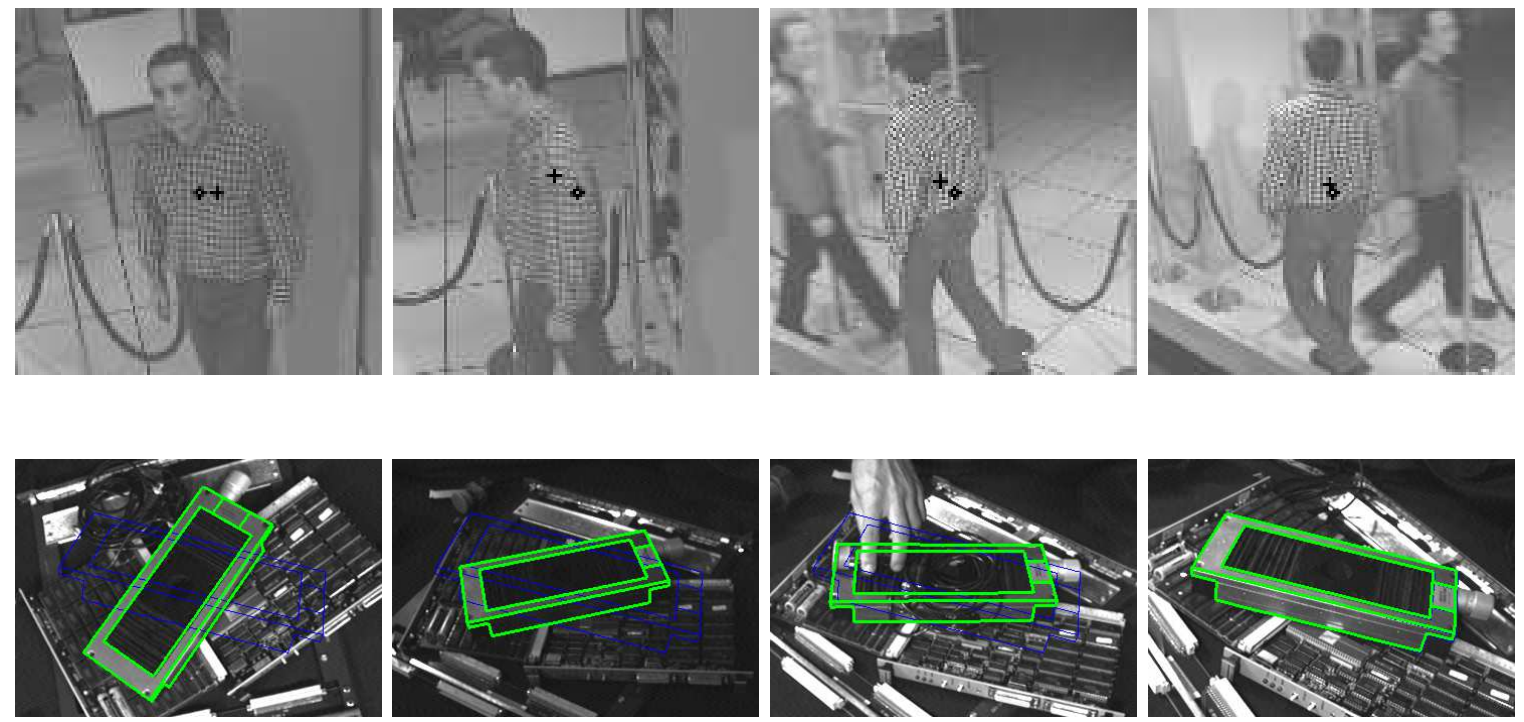

Fig. 1. Few images acquired during two visual servoing tasks: on the top, pedestrian tracking using a pan-tilt camera; on the bottom, controlling the 6 degrees of freedom of an eye-in-hand system so that an object appears at a particular position in the image (shown in blue). 


\section{Basic Theory}

Most if not all visual servoing tasks can be expressed as the regulation to zero of an error $\mathbf{e}(t)$ defined by

$$
\mathbf{e}(t)=\mathbf{s}(\mathbf{m}(\mathbf{r}(t)), \mathbf{a})-\mathbf{s}^{*}(t)
$$

The parameters in (1) are defined as follows (Chaumette et al. 2016): the vector $\mathbf{m}(\mathbf{r}(t))$ is a set of image measurements (e.g. the image coordinates of points, or the area, the center of gravity and other geometric characteristics of an object, ...). These image measurements depend on the pose $\mathbf{r}(t)$ between the camera and the environment, this pose varying with time $t$. They are used to compute a vector $\mathbf{s}(\mathbf{m}(\mathbf{r}(t))$, a) of visual features, in which $\mathbf{a}$ is a set of parameters that represent potential additional knowledge about the system (e.g. coarse camera intrinsic parameters or 3D model of objects). The vector $\mathbf{s}^{*}(t)$ contains the desired value of the features, which can be either constant in the case of a fixed goal, or varying if the task consists in following a specified trajectory.

Visual servoing schemes mainly differ in the way that the visual features are designed. As represented on Fig. 2, the two most classical approaches are named imagebased visual servoing (IBVS), in which $\mathbf{s}$ consists of a set of 2D parameters that are directly expressed in the image (Weiss et al. 1987; Espiau et al. 1992), and pose-based visual servoing (PBVS), in which $\mathbf{s}$ consists of a set of 3D parameters related to the pose between the camera and the target (Weiss et al. 1987; Wilson et al. 1996; Thuilot et al. 2002). In that case, the 3D parameters have to be estimated from the image measurements either through a pose estimation process using the knowledge of the 3D target model (Marchand et al. 2016), or through a triangulation process if a stereovision system is considered. Inside IBVS and PBVS approaches, many possibilities exist depending on the choice of the features. Each choice will induce a particular behavior of the system. There also exist hybrid approaches, named $21 / 2 \mathrm{D}$ visual servoing, which 
combine $2 \mathrm{D}$ and $3 \mathrm{D}$ parameters in $\mathbf{s}$ in order to benefit from the advantages of IBVS and PBVS while avoiding their respective drawbacks (Malis and Chaumette 2000).
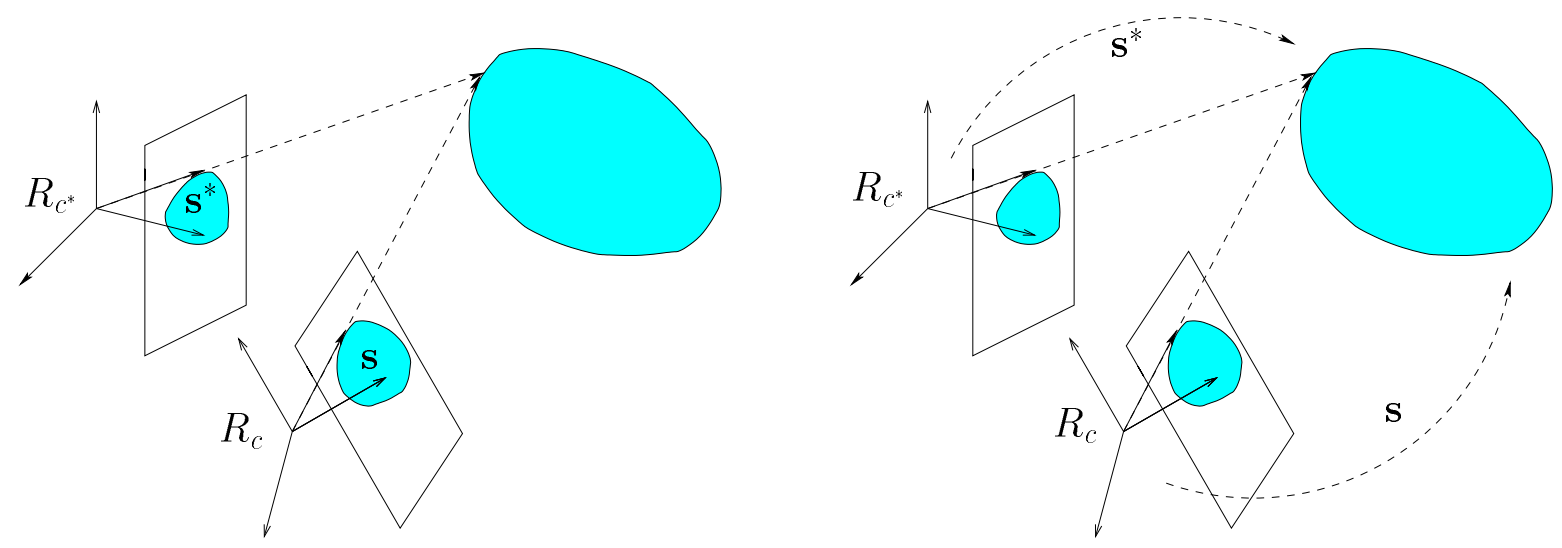

Fig. 2. If the goal is to move the camera from frame $R_{c}$ to the desired frame $R_{c^{*}}$, two main approaches are possible: IBVS on the left, where the features $\mathbf{s}$ and $\mathbf{s}^{*}$ are expressed in the image, and PBVS on the right, where the features $\mathbf{s}$ and $\mathbf{s}^{*}$ are related to the pose between the camera and the observed object.

\section{The features Jacobian}

The design of the control scheme is based on the link between the time variation $\dot{\mathbf{s}}$ of the features and the robot control inputs, which are usually the velocity $\dot{\mathbf{q}}$ of the robot joints. This relation is given by

$$
\dot{\mathbf{s}}=\mathbf{J}_{\mathbf{s}} \dot{\mathbf{q}}+\frac{\partial \mathbf{s}}{\partial t}
$$

where $\mathbf{J}_{\mathbf{s}}$ is the features Jacobian matrix, defined from the equation above similarly as the classical robot Jacobian. For an eye-in-hand system (see the left part of Fig. 3), the term $\frac{\partial \mathbf{s}}{\partial t}$ represents the time variation of $\mathbf{s}$ due to a potential object motion, while for an eye-to-hand system (see the right part of Fig. 3) it represents the time variation of $\mathbf{s}$ due to a potential sensor motion.

As for the features Jacobian, in the eye-in-hand configuration, it can be decomposed as (Chaumette et al. 2016) 

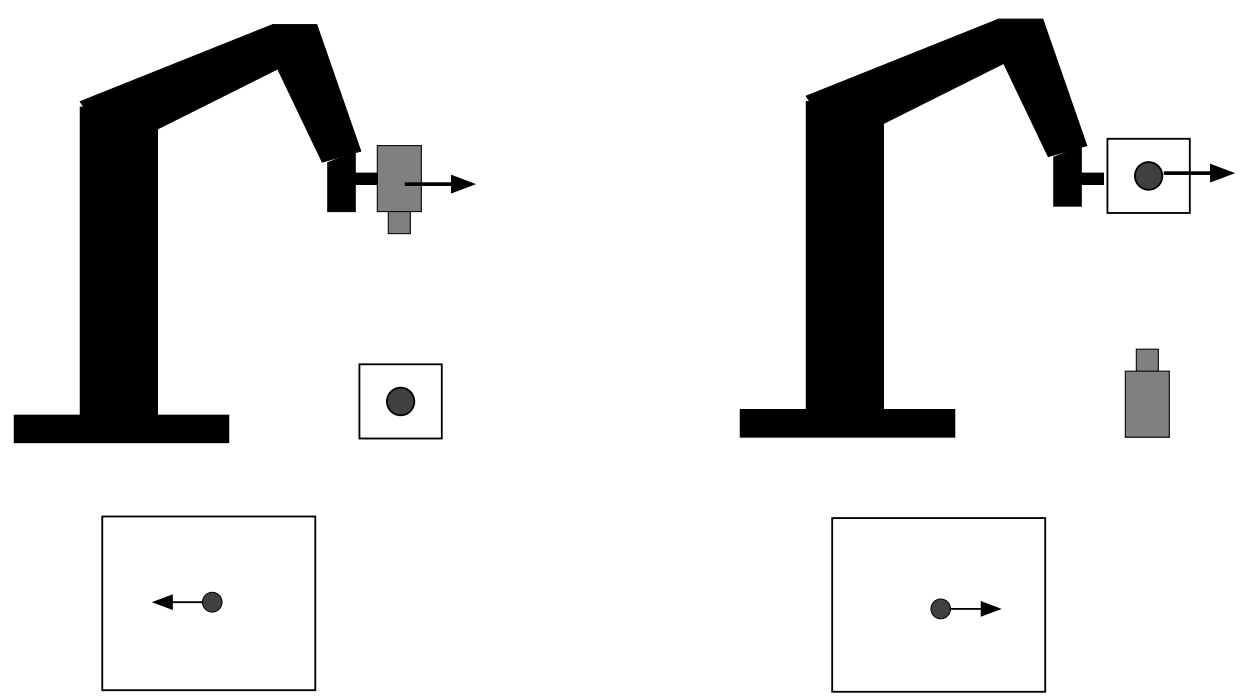

Fig. 3. In visual servoing, the vision sensor can be either mounted near the robot end-effector (eyein-hand configuration) or outside and observing the end-effector (eye-to-hand configuration). For the same robot motion, the motion produced in the image will be opposite from one configuration to the other.

$$
\mathbf{J}_{\mathbf{s}}=\mathbf{L}_{\mathbf{s}}{ }^{c} \mathbf{V}_{e} \mathbf{J}(\mathbf{q})
$$

where

- $\quad \mathbf{L}_{\mathbf{s}}$ is the interaction matrix of $\mathbf{s}$ defined such that

$$
\dot{\mathbf{s}}=\mathbf{L}_{\mathbf{s}} \mathbf{v}
$$

where $\mathbf{v} \in s e_{3}$ is the relative velocity between the camera and the environment expressed in the camera frame. More details on how to determine this matrix are given below.

- ${ }^{c} \mathbf{V}_{e}$ is the spatial motion transform matrix from the vision sensor to the endeffector. It is given by (Khalil and Dombre 2002)

$$
{ }^{c} \mathbf{V}_{e}=\left[\begin{array}{cc}
{ }^{c} \mathbf{R}_{e} & {\left[{ }^{c} \mathbf{t}_{e}\right]_{\times}{ }^{c} \mathbf{R}_{e}} \\
\mathbf{0} & { }^{c} \mathbf{R}_{e}
\end{array}\right]
$$

where ${ }^{c} \mathbf{R}_{e}$ and ${ }^{c} \mathbf{t}_{e}$ are respectively the rotation matrix and the translation vector between the sensor frame and the end-effector frame, and where $\left[{ }^{c} \mathbf{t}_{e}\right]_{\times}$is the skew symmetric matrix associated to ${ }^{c} \mathbf{t}_{e}$. Matrix ${ }^{c} \mathbf{V}_{e}$ is constant when the vision 
sensor is rigidly attached to the end-effector, which is usually the case. Thanks to the robustness of closed-loop control schemes with respect to calibration errors, a coarse approximation of ${ }^{c} \mathbf{R}_{e}$ and ${ }^{c} \mathbf{t}_{e}$ is generally sufficient in practice to serve as a satisfactory estimation of ${ }^{c} \mathbf{V}_{e}$ to be injected in the control law. If needed, an accurate estimation is possible through classical hand-eye calibration methods (Tsai and Lenz 1989).

- $\quad \mathbf{J}(\mathbf{q})$ is the robot Jacobian such that $\mathbf{v}_{e}=\mathbf{J}(\mathbf{q}) \dot{\mathbf{q}}$ where $\mathbf{v}_{e}$ is the robot endeffector velocity.

In the eye-to-hand configuration, the features Jacobian $\mathbf{J}_{\mathbf{s}}$ is composed of (Chaumette et al. 2016)

$$
\mathbf{J}_{\mathbf{s}}=-\mathbf{L}_{\mathbf{s}}{ }^{c} \mathbf{V}_{f}{ }^{f} \mathbf{V}_{e} \mathbf{J}(\mathbf{q})
$$

where

- $\quad{ }^{f} \mathbf{V}_{e}$ is the spatial motion transform matrix from the robot reference frame to the end-effector frame. It is known from the robot kinematics model.

- ${ }^{c} \mathbf{V}_{f}$ is the spatial motion transform matrix from the camera frame to the reference frame. It is constant as long as the camera does not move. In that case, similarly as for the eye-in-hand configuration, a coarse approximation of ${ }^{c} \mathbf{R}_{f}$ and ${ }^{c} \mathbf{t}_{f}$ is usually sufficient.

\section{The interaction matrix}

A lot of works have concerned the modeling of various visual features $\mathbf{s}$ and the determination of the analytical form of their interaction matrix $\mathbf{L}_{\mathbf{s}}$. To give just an example, in the case of an image point with normalized Cartesian coordinates $\mathbf{x}=(x, y)$ and whose 3D corresponding point has depth $Z$ in the camera frame, the interaction matrix $\mathbf{L}_{\mathbf{x}}$ of $\mathbf{x}$ is given by (Espiau et al. 1992) 


$$
\mathbf{L}_{\mathbf{x}}=\left[\begin{array}{cccccc}
-1 / Z & 0 & x / Z & x y & -\left(1+x^{2}\right) & y \\
0 & -1 / Z & y / Z & 1+y^{2} & -x y & -x
\end{array}\right]
$$

where the three first columns contain the elements related to the three components of the translational velocity, and where the three last columns contain the elements related to the three components of the rotational velocity.

By just changing the parameters representing the same image point, that is, by using the cylindrical coordinates defined by $\gamma=(\rho, \theta)$ with $\rho=\sqrt{x^{2}+y^{2}}$ and $\theta=\operatorname{Arctan}(y / x)$, the interaction matrix of these parameters has a completely different form (Iwatsuki and Okiyama 2005):

$$
\mathbf{L}_{\boldsymbol{\gamma}}=\left[\begin{array}{ccccc}
-\cos \theta / Z & -\sin \theta / Z & \rho / Z\left(1+\rho^{2}\right) \sin \theta-\left(1+\rho^{2}\right) \cos \theta & 0 \\
\sin \theta /(\rho Z)-\cos \theta /(\rho Z) & 0 & \cos \theta / \rho & \sin \theta / \rho & -1
\end{array}\right]
$$

This implies that using the Cartesian coordinates or the cylindrical coordinates as visual features will induce a different behavior, that is, a different trajectory of the point in the image and, consequently, a different robot trajector. The main objective in designing a visual servoing control scheme is thus to select the best set of visual features in terms of stability, global behavior (adequate trajectories both in the image plane and 3D space), and robustness to noise and to modeling and calibration errors from the task to be achieved, the environment observed, and the available image measurements. All these aspects can be studied from the interaction matrix of the potential visual features.

Currently, the analytical form of the interaction matrix is available for most basic features resulting from the perspective projection of simple geometrical primitives such as circles, spheres, and cylinders (Espiau et al. 1992). It is also available for image moments related to planar and almost-planar objects of any shape (Chaumette 2004), as well as for features selected from the epipolar geometry (Silveira and Malis 
2012), and, of course, also for coordinates of 3D points, parameters of 3D geometrical primitives, and pose parameters, assuming these features are perfectly estimated.

In the recent years, following the seminal works of Nayar et al. (1996) and Deguchi (2000), a new trend has concerned the use of direct image content as input of the control scheme (Han et al. 2010; Collewet and Marchand 2011). The main objective of these works is to avoid the extraction, tracking and matching of geometrical measurements, such as points of interest or edges, so that the system is extremely accurate and robust with respect to image processing errors. The basic idea is to consider the intensity of a set of pixels as visual features $(\mathbf{s}=\mathbf{I})$. From the classical assumption in computer vision stating that the intensity level of a moving point does not change (i.e. $I(\mathbf{x}, t)=I(\mathbf{x}+\mathbf{d} \mathbf{x}, t+d t))$, it is possible to determine the interaction matrix corresponding to the intensity level of a pixel:

$$
\mathbf{L}_{I}=-\left[\frac{\partial I}{\partial x} \frac{\partial I}{\partial y}\right] \mathbf{L}_{\mathbf{x}}
$$

where $\left[\frac{\partial I}{\partial x} \frac{\partial I}{\partial y}\right]$ is the spatial gradient of the intensity along the $x$ and $y$ directions. Proceeding so leads to control a highly nonlinear system, with the drawback of a relatively small convergence domain, and, in general, not expected robot trajectory, plus the potential issue of robustness with respect to lighting variations. That is why the idea of direct photometric visual servoing has been expended by either considering other objective functions than $\left\|\mathbf{I}-\mathbf{I}^{*}\right\|$, such as the mutual information between the current and desired images (Dame and Marchand 2011), or other global image representations (Duflot et al. 2019; Crombez et al. 2019), or by designing photo-geometric visual features (Bakthavatchalam and Chaumette 2018).

All the works mentioned above have considered a classical vision sensor modeled by a perspective projection. It is possible to generalize the approach to any sort of sensors, such as omnidirectional cameras (Hadj-Abdelkader et al. 2008; Caron et al. 2013), RGB-D sensors (Teulière and Marchand 2014), the coupling between a camera 
and structured light (Motyl et al. 1992, Pagès et al. 2006), and even 2D echographic probes (Mebarki et al. 2010). A large variety of visual features is thus available for many vision sensors.

Finally, methods also exist to estimate off-line or online a numerical value of the interaction matrix, by using neural networks for instance (Suh 1993, Wells et al. 1996), or the Broyden update (Koh and Hosoda 1994, Jägersand et al. 1997). These methods are useful when the analytical form of the interaction matrix cannot be determined, but any a priori analysis of the properties of the system is unfortunately impossible.

\section{Control}

Once the modeling step has been performed, the design of the control scheme can be quite simple for holonomic robots. The most basic control scheme has the following form (Chaumette et al. 2016)

$$
\dot{\mathbf{q}}=-\lambda{\widehat{\mathbf{J}_{\mathbf{s}}}}^{+} \mathbf{e}+\widehat{\mathbf{J}}_{\mathbf{s}}^{+} \frac{\partial \mathbf{s}^{*}}{\partial t}-\widehat{\mathbf{J}}_{\mathbf{s}}+\frac{\widehat{\partial \mathbf{s}}}{\partial t}
$$

where, in the first feedback term, $\mathbf{e}=\mathbf{s}-\mathbf{s}^{*}$ as defined in Eq. (1), $\lambda$ is a positive (possibly varying) gain tuning the time-to-convergence of the system, and ${\widehat{\mathbf{J}_{\mathbf{s}}}}^{+}$is the MoorePenrose pseudoinverse of an approximation or an estimation of the features Jacobian. The exact value of all its elements is indeed generally unknown since it depends of the intrinsic and extrinsic camera parameters, as well as of some 3D parameters such as the depth of the point in Eqs. (7) and (8). Methods for estimating these 3D parameters exist, either using the knowledge of the robot motion (De Luca et al. 2008), or the knowledge of the 3D object model when it is available (Marchand et al. 2016), or, up to a scalar factor, from partial pose estimation using the properties of the epipolar geometry between the current and the desired images (Malis and Chaumette 2000).

The second term of the control scheme anticipates for the variation of $\mathbf{s}^{*}$ in the case of a varying desired value. The third term compensates as much as possible a 
possible target motion in the eye-in-hand case and a possible camera motion in the eyeto-hand case. They are both null in the case of a fixed desired value and a motionless target or camera. They serve as feedforward terms for removing the tracking error in the other cases (Corke and Good 1996).

Following the Lyapunov theory, the stability of the system can be studied (Chaumette et al. 2016). Generally, visual servoing schemes can be demonstrated to be locally asymptotically stable (i.e. the robot will converge if it starts from a local neighborhood of the desired pose) if the errors introduced in $\widehat{\mathbf{J}_{\mathbf{s}}}$ are not too strong. Some particular visual servoing schemes can be demonstrated to be globally asymptotically stable (i.e. the robot will converge whatever its initial pose) under similar conditions. This is for instance the case for the pan-tilt camera control depicted on Fig. 1, for PBVS assuming the 3D parameters involved are perfectly estimated, and for well-designed IBVS schemes.

Finally, when the visual features do not constrain all the DoF, it is possible to combine the visual task with supplementary tasks such as, for instance, joint limits avoidance or the visibility constraint (to be sure that the target considered will always remain in the camera field of view). In that case, the redundancy framework (Nakamura et al. 1987) can be applied and the new error to be regulated to zero has the following form:

$$
\mathbf{e}_{n}=\widehat{\mathbf{J}}_{\mathbf{s}}^{+} \mathbf{e}+\left(\mathbf{I}-\widehat{\mathbf{J}}_{\mathbf{s}}^{+} \widehat{\mathbf{J}_{\mathbf{s}}}\right) \mathbf{e}_{2}
$$

where $\left(\mathbf{I}-{\widehat{\mathbf{J}_{\mathbf{s}}}}^{+} \widehat{\mathbf{J}_{\mathbf{s}}}\right)$ is a projection operator on the null space of the visual task e so that the supplementary task $\mathbf{e}_{2}$ will be achieved at best under the constraint that it does not perturb the visual task. A similar control scheme to (10) is now given by

$$
\dot{\mathbf{q}}=-\lambda \mathbf{e}_{n}-\frac{\widehat{\partial \mathbf{e}_{n}}}{\partial t}
$$


This scheme has for instance been applied for the first example depicted on Fig. 4 where the rotational motion of the mobile robot is controlled by vision while its translational motion is controlled by the odometry to move at a constant velocity.

Any other more advanced control strategy can be applied such as optimal control (Nelson and Khosla 1995, Hashimoto et al. 1996), coupling path planning and visual servoing (Mezouar and Chaumette 2002, Chesi 2009, Kazemi et al. 2013), model predictive control (Ginhoux et al. 2005, Allibert et al. 2010), or quadratic programming (Agravante et al. 2016) when visual tasks and visual constraints have to be simultaneously handled with other tasks and constraints. Particular care has to be considered for underactuated and nonholonomic systems for which adequate control laws have to be designed (Hamel and Mahony 2002; Mebarki et al. 2015; Mariottini et al. 2007; Lopez-Nicolas et al. 2010).

\section{Applications}

Potential applications of visual servoing are numerous. It can be used as soon as a vision sensor is available and a task is assigned to a dynamic system. A non-exhaustive list of examples is (see also Fig. 4)

- $\quad$ the control of a pan-tilt-zoom camera, as illustrated in Figure 1 for the pan-tilt case;

- $\quad$ grasping using a robot arm;

- $\quad$ locomotion and dexterous manipulation with a humanoid robot;

- $\quad$ micro- or nano-manipulation of MEMS or biological cells;

- $\quad$ pipe inspection by an underwater autonomous vehicle;

- $\quad$ autonomous navigation of a mobile robot in indoor or outdoor environment;

- $\quad$ aircraft landing; 
- autonomous satellite rendezvous;

- biopsy using ultrasound probes or heart motion compensation in medical robotics.

- virtual cinematography in animation.
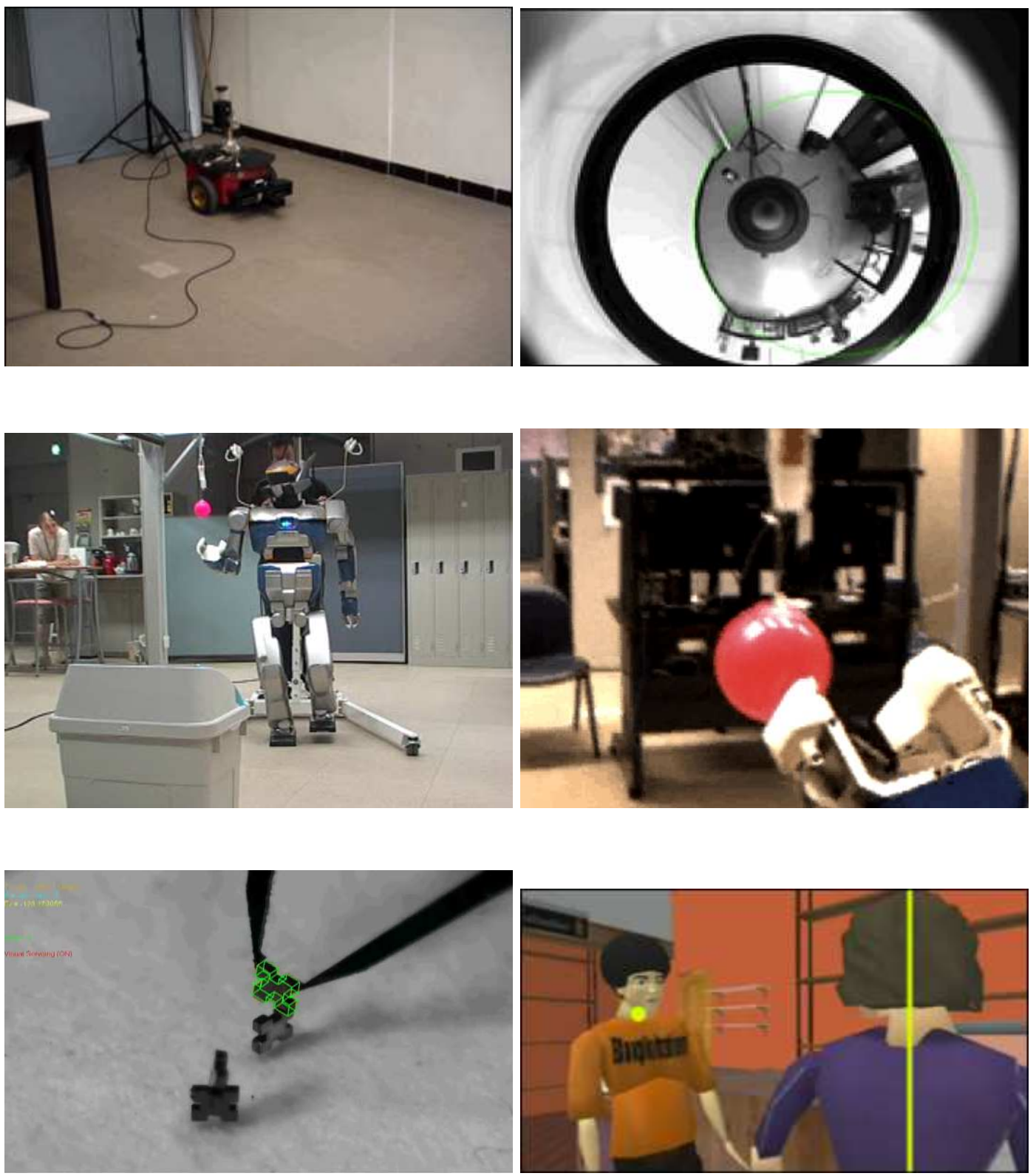

Fig. 4. Few applications of visual servoing: navigation of a mobile robot to follow a wall using an omnidirectional vision sensor (top line), grasping a ball with a humanoid robot (middle line), assembly of MEMS and film of a dialogue within the constraints of a script in animation (bottom line). 


\section{Summary and Future Directions}

Visual servoing is a mature area. It is basically a nonlinear control problem for which numerous modeling works have been achieved to design visual features so that the control problem is transformed as much as possible to a linear control problem. On one hand, improvements on this topic are still expected for instantiating this general approach to particular applications. On the other hand, designing new control strategies is another direction for improvements, especially when supplementary data coming from other sensors (force, tactile, proximity sensors) are available. Finally, the current expansion of deep learning may rejuvenate the field (Levine et al. 2016; Bateux et al. 2018; Pandya et al. 2019), especially for the dense direct methods that use the same input and end-to-end approach.

\section{Cross References}

- Robot Motion Control

- $\quad$ Image based Robot Control

- 2.5D Vision Based Estimation and Control

- $\quad$ Image-Based Formation Control of Mobile Robots

- Intermittent Image-based control

\section{Recommended Reading}

In addition to the references already cited in text, including the classical tutorials by Hutchinson et al. (1996) and Chaumette el al. (2016), the book by Corke (2011) and the collection of papers by Hashimoto (1993), Kriegman et al. (1998) and Chesi et al. (2010) provide a good overview of past works in the field. 


\section{References}

1. Agravante DJ, Claudio G, Spindler F, Chaumette F (2016). Visual servoing in an optimization framework for the whole-body control of humanoid robots. IEEE Robotics and Automation Letters, 2(2):608-615.

2. Allibert G, Courtial E, Chaumette F (2010). Predictive control for constrained image-based visual servoing. IEEE Transactions on Robotics, 26(5):933-939.

3. Bakthavatchalam M, Tahri O, Chaumette F (2018). A direct dense visual servoing approach using photometric moments. IEEE Transactions on Robotics, 34(5):1226-1239.

4. Bateux Q, Marchand E, Leitner J, Chaumette F, Corke P (2018). Training deep neural networks for visual servoing. IEEE Int. Conf. on Robotics and Automation (ICRA'18), pp. 3307-3314.

5. Caron G, Marchand E, Mouaddib EM (2013). Photometric visual servoing for omnidirectional cameras. Autonomous Robots, 35(2):177-193.

6. Chaumette F (2004). Image moments: a general and useful set of features for visual servoing. IEEE Transactions on Robotics, 20(4):713-723.

7. Chaumette F, Hutchinson S, Corke P (2016). Visual servoing. In Handbook of Robotics, 2nd edition, Chapter 34, pp. 841-866, Springer.

8. Chesi G, Hashimoto K (eds) (2010). Visual servoing via advanced numerical methods. LNCIS, vol. 401. Springer, Berlin.

9. Chesi G (2009). Visual servoing path planning via homogeneous forms and LMI optimizations. IEEE Transactions on Robotics, 25(2):281-291.

10. Collewet C, Marchand E (2011). Photometric visual servoing. IEEE Transactions on Robotics, $27(4): 828-834$

11. Corke P, Good M (1996). Dynamic effects in visual closed-loop systems. IEEE Transactions on Robotics and Automation, 12(5):671-683.

12. Corke P (2011). Robotics, Vision and Control. Springer Tracts in Advanced Robotics, vol. 73 Springer, Berlin.

13. Crombez N, Mouaddib EM, Caron G, Chaumette F (2019). Visual servoing with photometric Gaussian mixtures as dense features. IEEE Transactions on Robotics, 35(1):49-63.

14. Dame A, Marchand E (2011). Mutual information-based visual servoing. IEEE Transactions on Robotics, 27(5):958-969. 
15. Deguchi K (2000). A direct interpretation of dynamic images with camera and object motions for vision guided robot control, International Journal of Computer Vision, 37(1):7-20.

16. De Luca A, Oriolo G, Robuffo Giordano P (2008). Feature depth observation for image-based visual servoing: Theory and experiments, International Journal of Robotics Research, 38(4):422450. $27(10): 1093-1116$.

17. Duflot LA, Reisenhofer R, Tamadazte B, Andreff N, Krupa A (2019). Wavelet and shearlet-baed image representations for visual servoing, International Journal of Robotics Research, 38(4):422450.

18. Espiau B, Chaumette F, Rives P (1992). A new approach to visual servoing in robotics. IEEE Transactions on Robotics and Automation, 8(3):313-326.

19. Ginhoux R, Gangloff J, de Mathelin M, Soler L, Sanchez MA, Marescaux J (2005). Active filtering of physiological motion in robotized surgery using predictive control. IEEE Transactions on Robotics, 21(1):67-79

20. Hadj-Abdelkader H, Mezouar Y, Martinet P, Chaumette F (2008). Catadioptric visual servoing from 3D straight lines. IEEE Transactions on Robotics, 24(3):652-665.

21. Hamel T, Mahony R (2002). Visual servoing of an under-actuated dynamic rigid-body system: An image-based approach. IEEE Transactions on Robotics and Automation, 18(2):187-198.

22. Han S, Censi A, Straw A, Murray R (2010). A bio-plausible design for visual pose stabilization. Int. Conf. on Intelligent Robots and Systems (IROS'10), pp. 5679-5686.

23. Hashimoto K (ed) (1993). Visual servoing: real-time control of robot manipulators based on visual sensory feedback. World Scientific, Singapore.

24. Hashimoto K, Ebine T, Kimura H (1996). Visual servoing with hand-eye manipulator - Optimal control approach. IEEE Transactions on Robotics and Automation, 12(5):766-774.

25. Hosoda K, Asada M (1994). Versatile visual servoing without knowledge of true jacobian, IEEE/RSJ Int. Conf. on Intelligent Robots and Systems (IROS'94) pp. 186-193.

26. Hutchinson S, Hager G, Corke P (1996). A tutorial on visual servo control. IEEE Transactions on Robotics and Automation, 12(5): 651-670.

27. Iwatsuki M, Okiyama N (2005). A new formulation of visual servoing based on cylindrical coordinate system. IEEE Transactions on Robotics and Automation, 21(2):266-273.

28. Jägersand M, Fuentes O, Nelson R (1997). Experimental evaluation of uncalibrated visual servoing for precision manipulation, IEEE Int. Conf. on Robotics and Automation (ICRA'97), pp. 2874- 
2880.

29. Kazemi M, Gupta K, Mehrandezh M (2013). Randomized kinodynamic planning for robust visual servoing. IEEE Transactions on Robotics, 29(5):1197-1211.

30. Khalil W, Dombre E (2002). Modeling, Identification, and Control of Robots. CRC Press.

31. Kriegman D, Hager G, Morse S (eds) (1998) The confluence of vision and control. LNCIS, vol. 237. Springer, London.

32. Levine S, Finn C, Darrell T, Abbeel P (2016). End-to-end training of deep visuomotor policies. Journal of Machine Learning Research, 17(1):1334-1373.

33. Lopez-Nicolas G, Guerrero JJ, Sagues C (2010). Visual control through the trifocal tensor for nonholonomic robots. Robotics and Autonomous Systems, 58(2):216-226.

34. Malis E, Chaumette F (2000). 2-1/2D visual servoing with respect to unknown objects through a new estimation scheme of camera displacement. International Journal of Computer Vision, $37(1): 79-97$.

35. Marchand E, Spindler F, Chaumette F (2005). ViSP for visual servoing: a generic software platform with a wide class of robot control skills. IEEE Robotics and Automation Magazine, $12(4): 40-52$.

36. Marchand E, Uchiyama H, Spindler F (2016). Pose estimation for augmented reality: a hands-on survey. IEEE Transactions on Visualization and Computer Graphics, 22(12):2633-2651.

37. Mariottini GL, Oriolo G, Prattichizo D (2007). Image-based visual servoing for nonholonomic mobile robots using epipolar geometry, IEEE Transactions on Robotics, 23(1):87-100.

38. Mebarki R, Krupa A, Chaumette F (2010). 2D ultrasound probe complete guidance by visual servoing using image moments. IEEE Transactions on Robotics, 26(2):296-306.

39. Mebarki R, Lippiello V, Siciliano B (2015). Nonlinear visual control of unmanned aerial vehicles in GPS-denied environments. IEEE Transactions on Robotics, 31(4):1004-1017.

40. Mezouar Y, Chaumette F (2002). Path planning for robust image-based control. IEEE Transactions on Robotics, 22(10):781-804.

41. Motyl G, Chaumette F, Gallice J (1992). Coupling a camera and laser stripe in sensor based control. Second Int. Symp. on Measurement and Control in Robotics, pp. 685-692.

42. Nakamura Y, Hanafusa H, Yoshikawa T (1987). Task-priority based redundancy control of robot manipulators. International Journal of Robotics Research, 6(2):3-15 
43. Nayar S, Nene S, Murase H (1996). Subspace methods for robot vision. IEEE Transactions on Robotics and Automation, 12(5):750-758.

44. Nelson B, Khosla P (1995). Strategies for increasing the tracking region of an eye-in-hand system by singularity and joint limit avoidance. International Journal of Robotics Research, 14(3):225269.

45. Pagès J, Collewet C, Chaumette F, Salvi J (2006). Optimizing plane-to-plane positioning tasks by image-based visual servoing and structured light. IEEE Transactions on Robotics, 22(5):10001010.

46. Pandya H, Gaud A, Kumar G, Madhava Krishna K (2019). Instance invariant visual servoing framework for part-aware autonomous vehicle inspection using MAVs. Journal of Field Robotics, $36(5): 892-918$.

47. Silveira G, Malis E (2012). Direct visual servoing: Vision-based estimation and control using only nonmetric information. IEEE Transactions on Robotics, 28(4):974-980.

48. Suh I (1993). Visual servoing of robot manipulators by fuzzy membership function based neural networks. In: Visual Servoing, Vol 7 of World Scientific Series on Robotics and Automated Systems, pp. 285-315.

49. Teulière C, Marchand E (2014). A dense and direct approach to visual servoing using depth maps. IEEE Transactions on Robotics, 30(5):1242-1249.

50. Thuilot B, Martinet P, Cordesses L, Gallice J (2002). Position based visual servoing: Keeping the object in the field of vision. IEEE Int. Conf. on Robotics and Automation (ICRA'02), pp. 16241629.

51. Tsai R, Lenz R (1989). A new technique for fully autonomous and efficient 3D robotics hand-eye calibration. IEEE Transactions on Robotics and Automation, 5(3): 345-358.

52. Weiss L, Sanderson A, Neuman C (1987). Dynamic sensor-based control of robots with visual feedback. IEEE Journal on Robotics and Automation, 3(5): 404-417.

53. Wells G, Venaille C, Torras C (1996). Vision-based robot positioning using neural networks. Image and Vision Computing, 14(10):715-732.

54. Wilson W, Hulls C, Bell G (1996). Relative end-effector control using Cartesian position-based visual servoing. IEEE Transactions on Robotics and Automation, 12(5): 684-696. 